\title{
Acute Feasibility of Neuromuscular Electrical Stimulation in Severely Obese Patients with Obstructive Sleep Apnea Syndrome: A Pilot Study
}

\author{
Isabelle Vivodtzev, ${ }^{1}$ Nicola A. Maffiuletti, ${ }^{2}$ Anne-Laure Borel, ${ }^{1}$ Angélique Grangier, ${ }^{1}$ \\ Bernard Wuyam, ${ }^{1}$ Renaud Tamisier, ${ }^{1}$ and Jean-Louis Pépin ${ }^{1}$ \\ ${ }^{1}$ Université Grenoble Alpes, HP2 Laboratory, Inserm U1042, Grenoble University Hospital, CS 20217, 38045 Grenoble Cedex 9, France \\ ${ }^{2}$ Human Performance Lab, Schulthess Clinic, Zurich, Lengghalde 2, 8008 Zurich, Switzerland
}

Correspondence should be addressed to Isabelle Vivodtzev; ivivodtzev@chu-grenoble.fr

Received 9 October 2016; Revised 6 December 2016; Accepted 22 December 2016; Published 17 January 2017

Academic Editor: Mitja Lainscak

Copyright (C) 2017 Isabelle Vivodtzev et al. This is an open access article distributed under the Creative Commons Attribution License, which permits unrestricted use, distribution, and reproduction in any medium, provided the original work is properly cited.

\begin{abstract}
Objective. Obesity and obstructive sleep apnea (OSA) are closely interconnected conditions both leading to high cardiovascular risk. Inactivity is frequent and physical activity programs remain difficult in these patients. We investigated the acute feasibility of two neuromuscular electrical stimulation (NMES) modalities in extremely inactive obese patients with OSA. Design. A randomized cross-over study, with two experimental sessions (one per condition: multipath NMES versus conventional NMES). Setting. Outpatient research hospital. Subjects. Twelve patients with obesity, already treated for OSA. Interventions. No intervention. Measures. Feasibility outcomes included NMES current intensity, knee extension force evoked by NMES, and self-reported discomfort. Results. We found higher current intensity, a trend to significantly higher evoked force and lower discomfort during multipath NMES versus conventional NMES, suggesting better tolerance to the former NMES modality. However, patients were rapidly limited in the potential of increasing current intensity of multipath NMES. Conclusion. Both NMES modalities were feasible and relatively well tolerated by obese patients with OSA, even if multipath NMES showed a better muscle response/discomfort ratio than conventional NMES. There is an urgent need for a proof-of-concept study and interventional randomized controlled trials comparing NMES therapy versus current care to justify its utilization in obese and apneic patients with low physical activity levels.
\end{abstract}

\section{Introduction}

Obesity is a growing public health problem leading to increased cardiovascular risk [1]. Obstructive sleep apnea syndrome (OSA), leading to intermittent hypoxia and sleep fragmentation, is highly prevalent in patients with obesity [2] and synergistically participates towards the occurrence of cardiometabolic comorbidities [3]. Furthermore, inactivity, another common risk factor for multimorbidity, is frequent in overweight or obese patients with OSA [4], putting these patients at high risk of cardiovascular diseases.

The inability to withstand physical effort is central to the symptoms of both obesity and OSA. Obesity is associated with reduced respiratory compliance [5], lower cardiac performance at exercise due to cardiac lipotoxicity [6], and frequent osteoarthritis [7]. On the other hand, OSA is associated with a modified hemodynamic response during exercise [8], daily somnolence, and impairment of muscle (oxidative) energy metabolism [9], independently of the physical activity level of the patients. Effective treatment of OSA by continuous positive airway pressure (CPAP) or noninvasive ventilation (NIV) may represent an important target for improving cardiometabolic risk. However, both fail to reduce metabolic or inflammatory markers in obese patients with OSA [10] or obesity hypoventilation syndrome [11] and have no significant effect on spontaneous physical activity [12]. Spontaneous physical activity in these patients is very low, particularly in terms of intensity level [13]. This emphasizes the need to offer a combination of multiple modalities of treatment to this specific population of obese patients with treated OSA. Lifestyle interventions and training programs using various modalities of exercise to reduce inactivity have 
been extensively implemented. For many patients, however, the implementation of physical activity programs remains difficult for several reasons including the level of disability (further complicated by orthopedic disorders) and psychosocial causes (the scrutiny of others, depression, and the lack of specific equipment dedicated to the morbidly obese). Finally, sleep apnea is known to attenuate the effects of a lifestyle intervention program in men with visceral obesity [14].

Transcutaneous neuromuscular electrical stimulation (NMES) was originally introduced as a treatment modality to prevent deconditioning associated with immobilization, particularly for orthopedic patients [15]. As it has minimal ventilatory requirements, NMES is currently emerging as a promising alternative to general physical reconditioning in patients with advanced respiratory diseases who are either unable or unwilling to attend formal rehabilitation programs or to undertake reasonable spontaneous physical activity. NMES programs have been shown to enhance skeletal muscle mass and strength $(+20 \%$ to $+50 \%)$, particularly for the quadriceps muscle [16]. This would thus allow patients to better carry their body weight and minimize breathlessness during daily activities, which can in turn improve exercise performance and quality of life [16]. From a metabolic point of view, we and others have previously reported changes at the muscle molecular level in patients with chronic obstructive pulmonary disease (COPD) $[17,18]$. NMES therapy significantly activated the (IGF-1/AKt) insulin signaling pathway in the stimulated quadriceps muscle [17], and the increase in muscle mass was comparable to that observed with voluntary exercise training [19]. Furthermore, NMES training may improve blood markers of insulin sensitivity in diabetes mellitus and obesity $[20,21]$. These studies collectively suggest a potential protective metabolic effect of NMES therapy in overweight or obese patients although the exact molecular mechanisms remain to be determined.

A novel NMES paradigm has recently been introducedmultipath NMES - which has been shown to maximize quadriceps muscle recruitment while minimizing the discomfort associated with the exogenous stimulation both in healthy subjects [22] and in overweight orthopedic patients [23]. Therefore, the objective of this pilot study was to investigate the acute feasibility of multipath NMES and conventional NMES of the quadriceps femoris muscle in obese patients already treated for OSA. To this aim, NMES current intensity, knee extension force evoked by NMES, and self-reported discomfort were systematically compared between the two NMES modalities, which were randomly administered on two separate experimental sessions (crossover design). We hypothesized that the use of multiple dynamically changing current pathways and large stimulating electrodes in multipath NMES would evoke stronger contractions (better recruitment) with less discomfort compared to conventional NMES [22].

\section{Methods}

\subsection{Participants}

2.1.1. Inclusion. Twelve consecutive patients of the sleep department medical consultation in Grenoble University Hospital (Grenoble, France) were included in the study.
TABLE 1: Patients' characteristics.

\begin{tabular}{lcc}
\hline Variables & Mean & SD \\
\hline Gender $(\mathrm{M} / \mathrm{F})$ & $7 / 5$ & \\
Age $($ years $)$ & 57 & 10 \\
BMI $\left(\mathrm{kg} / \mathrm{m}^{2}\right)$ & 40 & 5 \\
FFMI $\left(\mathrm{kg} / \mathrm{m}^{2}\right)$ & 22 & 5 \\
Waist circumference $(\mathrm{cm})$ & 113 & 15 \\
Hip circumference $(\mathrm{cm})$ & 121 & 5 \\
CPAP/NIV treatment & $8 / 4$ & \\
CPAP/NIV compliance (h/night) & 6 & 3 \\
FEV $(\mathrm{L} /$ min) & 2.3 & 0.8 \\
MVC force & & \\
$\quad$ Multipath NMES $(\mathrm{kg})$ & 43.0 & 28.4 \\
Conventional NMES $(\mathrm{kg})$ & 44.5 & 27.7 \\
\hline
\end{tabular}

BMI: body mass index; FFMI: fat-free mass index; CPAP: continuous positive airway pressure; NIV: noninvasive ventilation; $\mathrm{FEV}_{1}$ : forced expiratory volume in one second; MVC: maximal voluntary contraction; NMES: neuromuscular electrical stimulation.

Inclusion criteria were (1) adults with OSA based on apnea hypopnea index > 30/night at the time of diagnosis [24]; (2) being already treated for OSA by CPAP or NIV by more than 1 month with a compliance $>4$ h per night; (3) obesity $(35<$ $\mathrm{BMI}<45 \mathrm{~kg} / \mathrm{m}^{2}$ ); and (4) extremely low physical activity levels ( $<5000$ steps per day or less than $10 \mathrm{~min}$ walking/day). This trial was part of the ongoing longer-term study referenced as clinical trial NCT01820598. Ethical approval was obtained from the local ethical committee (CPP Sud Est V, France) and all the participants gave written informed consent.

2.1.2. Characteristics. Patients' characteristics are reported in Table 1 . The majority of patients were male (58\%), aged $57 \pm 10$ years; they had a body mass index (BMI) of $40 \pm 5 \mathrm{~kg} / \mathrm{m}^{2}$ and a fat-free mass index (FFMI) of $22 \pm 5 \mathrm{~kg} / \mathrm{m}^{2}$, as assessed by bioimpedance analysis (Bodystat ${ }^{\circledR} 1500$ MDD Body Composition Monitor). They were well compliant to their nocturnal OSA treatment (by CPAP or NIV). Patient comorbidities were type 2 diabetes mellitus (33\%), dyslipidemia (33\%), and hypertension (66\%). All patients were unfamiliar with NMES.

2.2. Experimental Procedures. Twelve participants completed two identical experimental sessions, except for the type of NMES-multipath NMES (Kneehab ${ }^{\circledR}$, BMR Ltd., Neurotech, Ireland) versus conventional NMES (Rehab 400 CefarCompex $^{\circledR}$, Scandinavia AB, Sweden)-that were randomly presented in a cross-over design (Figure 1). Experimenters and participants were both aware of which NMES modality was administered. Both NMES sessions lasted $20 \mathrm{~min}$ and were completed at the maximal tolerable intensity with the same stimulation frequency $(50 \mathrm{~Hz})$ and pulse duration $(400 \mu \mathrm{s})$. The experimental sessions were separated by at least $48 \mathrm{~h}$ and completed at the same time of day. Importantly, to mimic the real life condition at home, the patient increased by him-/herself the current intensity to his/her own maximal tolerable threshold throughout the experimental sessions. 


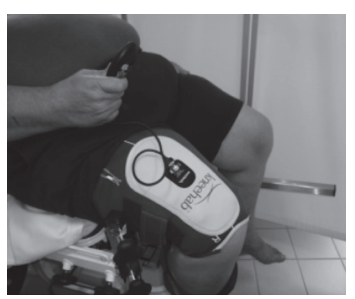

Multipath NMES

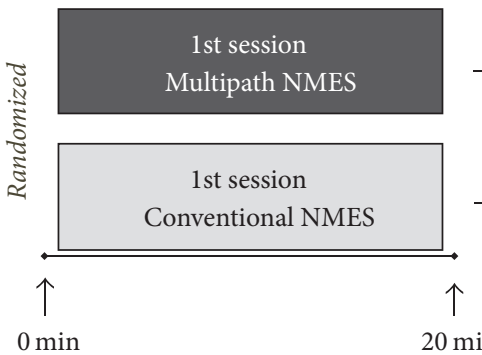

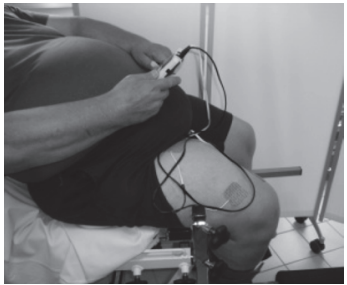

Conventional NMES

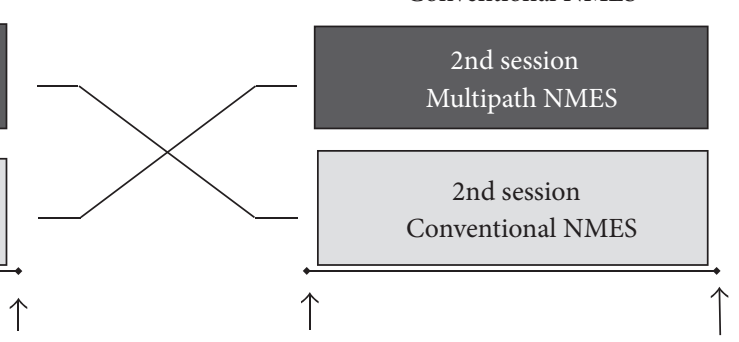
(i) Maximal current intensity
(ii) NMES-evoked muscle force
(i) Maximal current intensity
(iii) Leg discomfort (VAS)
(ii) NMES-evoked muscle force
(iii) Leg discomfort (VAS)

$20 \mathrm{~min}$

FIgURE 1: Overview of the experimental design: two 20 min sessions of NMES (one per condition: multipath NMES versus conventional NMES) were completed in a random order, with a cross-over design. In both conditions, stimulation frequency was $50 \mathrm{~Hz}$, pulse duration was $400 \mu \mathrm{s}$, and current intensity was self-determined at the maximal tolerable level. The two experimental sessions were separated by at least $48 \mathrm{~h}$.

2.2.1. Multipath NMES. Multipath NMES was delivered with a two-channel Kneehab XP device (Bio-Medical Research, Galway, Ireland), which consists of a stimulation unit connected to a garment that wraps around the thigh and incorporates four large self-adhesive pregelled electrodes (10 $\times 20 ; 3 \times 18 ; 10 \times 7.5$; and $7 \times 14 \mathrm{~cm})$.

2.2.2. Conventional NMES. Conventional NMES was delivered with a portable and programmable stimulator unit (Compex 3, Compex Médical SA, Ecublens, Switzerland) connected to three self-adhesive pregelled electrodes (two active channels). Two $5 \times 5 \mathrm{~cm}$ electrodes were positioned on the belly of the vastus lateralis and vastus medialis muscles. A large $(5 \times 10 \mathrm{~cm})$ self-adhesive electrode was fixed on the gluteal crease to close the stimulation current loop.

2.3. Outcome Measures. The ability to increase NMES current intensity during a typical session (tolerance), the level of knee extension force evoked by NMES (as a percentage of the maximal voluntary contraction (MVC) force), and the level of discomfort associated with NMES were assessed in each condition.

2.3.1. NMES Current Intensity. Current intensity was monitored by the patients themselves to ensure real life conditions. Participants were asked to progressively increase NMES current intensity as much as they could all along the session to attain a maximal sustainable intensity at midsession (10 min). This corresponded to a current level that they could sustain the remaining $10 \mathrm{~min}$ as well as the days after the session with the assumption that they would have continued NMES.

\subsubsection{Maximal Voluntary Contraction Force and Force Evoked} by NMES. Isometric quadriceps MVC force was measured using a strain-gauge transducer (Sensy, 2712 model, Jumet, Belgium), a signal transducer, and specific software (ADInstruments PowerLab Systems), as previously described in our laboratory [13]. Patients were asked to sit on a custom-made bench with a $90^{\circ} \mathrm{knee}$ and hip flexions and to cross their arms over the chest; a strap was fixed over the waist to avoid strength overestimation. The strain gauge was posteriorly attached to the leg, $2-3 \mathrm{~cm}$ above the lateral malleolus. The highest force value of three reproducible contractions $(<10 \%$ variability) was considered as MVC force. MVC force was measured $20 \mathrm{~min}$ before each NMES session (multipath NMES and conventional NMES) and the corresponding data are reported in Table 1. Evoked force was then measured on the same bench with the same gauge during series of contractions evoked by NMES, approximately 15 min after the beginning of each NMES session. The highest value of three reproducible measurements was considered as the evoked force by NMES.

2.3.3. Discomfort. The level of discomfort associated with the application of NMES was assessed by a visual analogical scale (VAS) from 0 (no discomfort) to 10 (extreme/unsustainable discomfort), approximately $15 \mathrm{~min}$ after the beginning of each NMES session.

2.4. Analysis Methods. Continuous data were presented as mean and standard deviation (Table 1) or individuals' data with median and standard errors (Figure 2). For all tests, a significance level of 0.05 was used. Normality was checked 


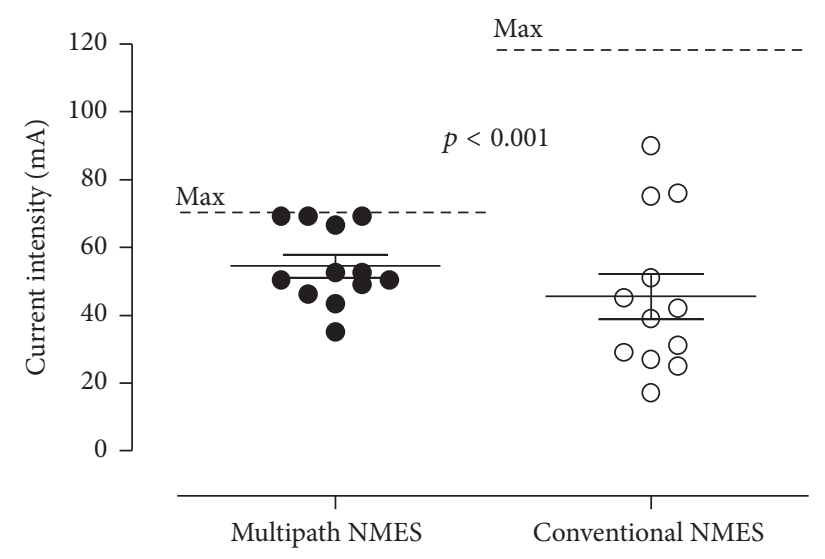

(a)

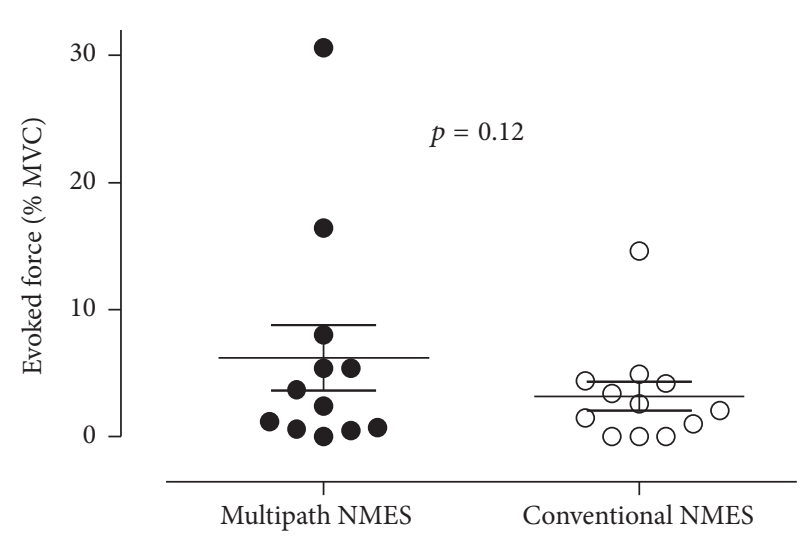

(b)

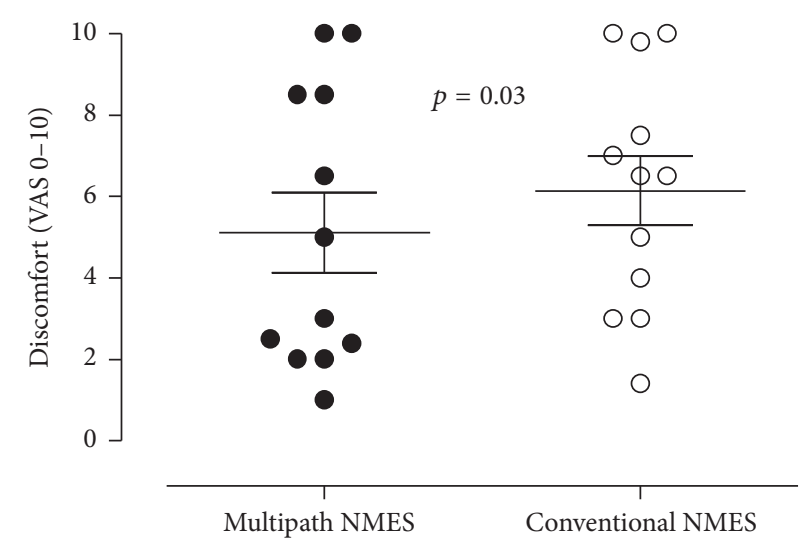

(c)

FIGURE 2: Acute responses to a single session of multipath versus conventional NMES in obese patients with OSA: comparison of individual values of (a) self-selected current intensity (dashed line: maximal current intensity delivered by each device); (b) NMES-evoked force; and (c) self-reported discomfort by stimulation modality (multipath versus conventional NMES, $n=12$ for both treatment sessions). Bars are median and standard errors.

using tests of Shapiro-Wilk and Skewness with a criteria of acceptance of $-1.0<$ Skewness score $<1.0$. The comparisons of the variables (current intensity, evoked force, and discomfort) between the two conditions (multipath versus conventional NMES) were made by paired $t$-test or Wilcoxon signed ranks test for paired data depending on the normality of the data. All statistical analyses were performed using SAS 9.4 software (SAS Institute Inc., Cary, NC). These results have been presented at the American Thoracic Society meeting in 2016 [25].

\section{Results}

3.1. Feasibility and Tolerance. All patients were able to complete the two NMES sessions with no adverse events. The highest current intensity reached during the session was $55 \pm$ 12 and $46 \pm 23 \mathrm{~mA}$ for multipath NMES and conventional NMES, respectively ( $p<0.001$; Figure 2(a)). The mean interval between $\mathrm{m}$-NMES and c-NMES for current intensity was $8.9 \pm 18.8 \mathrm{~mA}$ [min: -22.5 , max: $30.3 \mathrm{~mA}$ ].
3.2. Evoked Force by NMES. NMES-evoked force was $6.2 \pm$ 9.0\% MVC for multipath NMES versus $3.2 \pm 4.0 \%$ MVC for conventional NMES ( $p=0.12$; Figure 2(b)). $42 \%$ ( 5 out of 12 ) and $17 \%(2 / 12)$ of the patients achieved a knee extension force greater than $5 \%$ and $15 \% \mathrm{MVC}$, respectively, with multipath NMES versus only $17 \%(2 / 12)$ and $8 \%(1 / 12)$ for conventional NMES. The mean interval between m-NMES and c-NMES for evoked force was $3.0 \pm 8.5 \%$ MVC [min: -3.5 ; max: $30.6 \%$ MVC].

3.3. Discomfort. The level of discomfort was $5 \pm 3$ and $6 \pm 3$ for multipath and conventional NMES, respectively ( $p=0.03$; Figure 2(c)). This suggests a better tolerance to multipath NMES although patients were rapidly limited in the potential of increasing current intensity with this modality (see also Figure 2(a)), contrary to conventional NMES. The mean interval between m-NMES and c-NMES for the level of discomfort was $-1.0 \pm 1.7$ [min: -4.5 ; max: 1.0].

3.4. Subjective Preference. Once completed the two experimental sessions, 8 patients declared having preferred the 
multipath NMES modality, 1 patient declared having preferred the conventional NMES modality, and 3 patients had no preference.

\section{Discussion}

The acute application of both multipath and conventional NMES was feasible with good tolerance in obese patients with OSA and visible evoked quadriceps muscle contractions in half of them. Importantly, acceptable knee extension force levels could be successfully achieved in some but not all patients, particularly with multipath NMES. This result is highly relevant because it proves that existing NMES technologies allow the electrical current to penetrate the subcutaneous adipose tissue and to attain the muscle, thus inducing visible contractions even in severely obese patients.

There are limitations to NMES implementation in obese patients directly related to their body composition. Excessive amounts of subcutaneous adipose tissue represent a physiological barrier that is likely to affect the potential effectiveness of NMES therapy for obese patients because of the increased distance between the stimulating electrode and the axon terminals and also because body fat is a poor conductor of electricity [26]. Therefore, high current intensities are required to induce visible muscle contractions in obese individuals, and this is quite likely to activate nociceptors in addition to muscle fibers, thus limiting the application of NMES in this specific population. Thus, as expected, current intensity levels found in the present study were much higher than previously reported in other chronic diseases such as COPD or heart disease $[16,27]$. For a first session, the mean intensity level was about $45 \mathrm{~mA}$ as compared to 20 to $30 \mathrm{~mA}$ for similar NMES currents in COPD. The levels of force evoked by NMES were notably lower than those observed in healthy subjects at first NMES use [22], but actually close to those reported in other chronic diseases. In fact, with a mean of $6.2 \pm 9.0 \%$ MVC for multipath NMES (but up to $16.4 \%$ and $30.6 \%$ of MVC in two patients), the force levels obtained in this pilot study and particularly with the multipath system were similar to those observed at first NMES application in patients with COPD $[17,28]$. It is worth noting that an increase in current intensity is largely expected in the days following the very first NMES session, which could reach $>+10 \mathrm{~mA}$ for the first week [28]. Our current results are thus of importance since we demonstrated that it was possible to evoke visible muscle contractions in about half of the patients when initially exposed to NMES. In patients with COPD, we previously reported that, after a 6 -week NMES training, evoked force by NMES achieved a mean of $13 \pm 10 \%$ MVC [17]. Even low, this amount of evoked force by NMES allowed an increase in muscle strength by $20 \%$ but also in muscle cross-sectional area by $6 \%$ in mean in these patients. Those results were linked to changes at the molecular level which suggested a successful stimulation of muscle protein synthesis [17]. Importantly both in our previous study in COPD and in the present study, patients monitored intensity level by themselves, suggesting that patients will be able to maintain those intensities at home.
It is not surprising however that not all the patients were able to reach high NMES current intensities during a single session [28]. In our study, almost half of the patients did not reach 6\% MVC during their first NMES session. It is however likely that most of them will be able to double the current intensity in the first week of treatment [28]. The aptitude to tolerate high current intensities is extremely variable across patients, is individual specific [29], and depends on not only different uncontrollable factors such as skin and nerve sensitivity but also acceptance to discomfort [28]. Discomfort scores during the initial applications of NMES must be low to allow increasing current intensity during the whole treatment program, a condition for ensuring NMES effectiveness [17]. This aspect confirms the interest of multipath NMES for obese patients because of the lower discomfort with a trend for significantly higher evoked force, that is, a better muscle response/discomfort ratio as compared to conventional NMES. This is likely to explain why more patients subjectively preferred the multipath modality as compared to the conventional one (8 versus 1 out of 12 ).

4.1. Clinical Impact. Effective treatments of OSA for nocturnal respiratory troubles have been found to have little effect on cardiometabolic parameters in patients with OSA and likewise in obese patients who in addition cumulate metabolic comorbidities. In already CPAP treated patients, excluded for some reasons from usual exercise training program, combined strategies of care are necessary to counterbalance inactivity and muscle wasting. Until now, excessive amounts of subcutaneous adipose tissue have been considered as a physiological barrier affecting the potential effectiveness of NMES therapy in obese patients. With the recent clinical validation of the novel multipath NMES paradigm in an orthopedic population, which is able to maximize quadriceps muscle recruitment while minimizing the discomfort, we believe that NMES could be an interesting tool to increase the chance to maintain or even improve spontaneous physical activity in obese patients with OSA. Based on the result of the present study, we have initiated a larger, multicenter, and sham-controlled randomized trial to investigate the effect of m-NMES on spontaneous physical activity, functional parameters, and cardiometabolic parameters in severely obese patients with OSA.

Existing NMES devices show technical limitations (e.g., maximal current output, current distribution); thus they should be improved and adapted to the chronic disease population. Conventional NMES seems less appropriate for obese patients, due to limited muscle recruitment and high levels of discomfort. Multipath NMES is potentially appealing for this population also because of the excellent compliance for use at home (better than conventional NMES) [30], but the manufacturers should seriously consider the options to (1) increase the maximal current output from $70 \mathrm{~mA}$ to at least $100 \mathrm{~mA}$ to allow larger improvements in current intensity (and thus evoked force) within and between sessions and (2) extend the stimulation technology to muscle groups other than the quadriceps femoris (e.g., glutei, hamstrings) [21].

In conclusion, the acute application of NMES was feasible and well tolerated by obese patients with OSA and with a 
potential for molecular effects and muscle mass improvement (in case of repeated use), though it deserves to be proven in this population. There is an urgent need for a proofof-concept study and interventional randomized controlled trials comparing NMES therapy versus current care to justify its utilization in obese and already treated apneic patients with low physical activity levels.

\section{Competing Interests}

The authors declare that they have no competing interests.

\section{Acknowledgments}

This study was funded by endowment fund "Agir pour les Maladies Chroniques," La Tronche, France. The authors are grateful to Sonia Dias-Domingos for statistical analyses. They thank Alison Foote (Grenoble Clinical Research Center) for critically reading and editing of the manuscript.

\section{References}

[1] H. B. Hubert, M. Feinleib, P. M. McNamara, and W. P. Castelli, "Obesity as an independent risk factor for cardiovascular disease: a 26-year follow-up of participants in the Framingham Heart Study," Circulation, vol. 67, no. 5, pp. 968-977, 1983.

[2] P. E. Peppard, T. Young, M. Palta, J. Dempsey, and J. Skatrud, "Longitudinal study of moderate weight change and sleepdisordered breathing," The Journal of the American Medical Association, vol. 284, no. 23, pp. 3015-3021, 2000.

[3] J.-P. Baguet, G. Barone-Rochette, R. Tamisier, P. Levy, and J.-L. Pépin, "Mechanisms of cardiac dysfunction in obstructive sleep apnea," Nature Reviews Cardiology, vol. 9, no. 12, pp. 679-688, 2012.

[4] M. Mendelson, R. Tamisier, D. Laplaud et al., "Low physical activity is a determinant for elevated blood pressure in high cardiovascular risk obstructive sleep apnea," Respiratory Care, vol. 59, no. 8, pp. 1218-1227, 2014.

[5] S. W. Littleton, "Impact of obesity on respiratory function," Respirology, vol. 17, no. 1, pp. 43-49, 2012.

[6] L. R. Peterson, P. Herrero, K. B. Schechtman et al., "Effect of obesity and insulin resistance on myocardial substrate metabolism and efficiency in young women," Circulation, vol. 109, no. 18, pp. 2191-2196, 2004.

[7] A. E. Wluka, C. B. Lombard, and F. M. Cicuttini, "Tackling obesity in knee osteoarthritis," Nature Reviews Rheumatology, vol. 9, no. 4, pp. 225-235, 2013.

[8] L. Grote, J. Hedner, and J. H. Peter, "The heart rate response to exercise is blunted in patients with sleep-related breathing disorder," Cardiology, vol. 102, no. 2, pp. 93-99, 2004.

[9] D. Vanuxem, M. Badier, C. Guillot, S. Delpierre, F. Jahjah, and P. Vanuxem, "Impairment of muscle energy metabolism in patients with sleep apnoea syndrome," Respiratory Medicine, vol. 91, no. 9, pp. 551-557, 1997.

[10] I. Jullian-Desayes, M. Joyeux-Faure, R. Tamisier et al., "Impact of obstructive sleep apnea treatment by continuous positive airway pressure on cardiometabolic biomarkers: a systematic review from sham CPAP randomized controlled trials," Sleep Medicine Reviews, vol. 21, pp. 23-38, 2015.
[11] J.-C. Borel, R. Tamisier, J. Gonzalez-Bermejo et al., "Noninvasive ventilation in mild obesity hypoventilation syndrome: a randomized controlled trial," Chest, vol. 141, no. 3, pp. 692-702, 2012.

[12] S. D. West, M. Kohler, D. J. Nicoll, and J. R. Stradling, “The effect of continuous positive airway pressure treatment on physical activity in patients with obstructive sleep apnoea: a randomised controlled trial," Sleep Medicine, vol. 10, no. 9, pp. 1056-1058, 2009.

[13] I. Vivodtzev, M. Mendelson, M. Croteau et al., "Physiological correlates to spontaneous physical activity variability in obese patients with already treated sleep apnea syndrome," Sleep and Breathing, 2016.

[14] A.-L. Borel, X. Leblanc, N. Alméras et al., "Sleep apnoea attenuates the effects of a lifestyle intervention programme in men with visceral obesity," Thorax, vol. 67, no. 8, pp. 735-741, 2012.

[15] J. N. A. Gibson, K. Smith, and M. J. Rennie, "Prevention of disuse muscle atrophy by means of electrical stimulation: maintenance of protein synthesis," The Lancet, vol. 332, no. 8614, pp. 767-770, 1988.

[16] I. Vivodtzev, Y. Lacasse, and F. Maltais, "Neuromuscular electrical stimulation of the lower limbs in patients with chronic obstructive pulmonary disease," Journal of Cardiopulmonary Rehabilitation and Prevention, vol. 28, no. 2, pp. 79-91, 2008.

[17] I. Vivodtzev, R. Debigaré, P. Gagnon et al., "Functional and muscular effects of neuromuscular electrical stimulation in patients with severe COPD: a randomized clinical trial," Chest, vol. 141, no. 3, pp. 716-725, 2012.

[18] A. Abdellaoui, C. Préfaut, F. Gouzi et al., "Skeletal muscle effects of electrostimulation after COPD exacerbation: a pilot study," European Respiratory Journal, vol. 38, no. 4, pp. 781-788, 2011.

[19] M. I. Lewis, M. Fournier, T. W. Storer et al., "Skeletal muscle adaptations to testosterone and resistance training in men with COPD," Journal of Applied Physiology, vol. 103, no. 4, pp. 12991310, 2007.

[20] Y. Z. Feng, N. Nikolić, S. S. Bakke et al., "Myotubes from lean and severely obese subjects with and without type 2 diabetes respond differently to an in vitro model of exercise," American Journal of Physiology-Cell Physiology, vol. 308, no. 7, pp. C548C556, 2015.

[21] L. Crowe and B. Caulfield, "Aerobic neuromuscular electrical stimulation-an emerging technology to improve haemoglobin A1c in type 2 diabetes mellitus: results of a pilot study," $B M J$ Open, vol. 2, no. 3, Article ID e000219, 2012.

[22] N. A. Maffiuletti, I. Vivodtzev, M. A. Minetto, and N. Place, "A new paradigm of neuromuscular electrical stimulation for the quadriceps femoris muscle," European Journal of Applied Physiology, vol. 114, no. 6, pp. 1197-1205, 2014.

[23] C. Morf, V. Wellauer, N. C. Casartelli, and N. A. Maffiuletti, "Acute effects of multipath electrical stimulation in patients with total knee arthroplasty," Archives of Physical Medicine and Rehabilitation, vol. 96, no. 3, pp. 498-504, 2015.

[24] W. W. Flemons, D. Buysse, S. Redline et al., "Sleep-related breathing disorders in adults: recommendations for syndrome definition and measurement techniques in clinical research. The Report of an American Academy of Sleep Medicine Task Force," Sleep, vol. 22, no. 5, pp. 667-689, 1999.

[25] I. Vivodtzev, N. A. Maffiuletti, A. L. Borel et al., "Neuromuscular electrical stimulation in obese patients with Obstructive Sleep Apnea (OSA): a pilot study," American Journal of Respiratory and Critical Care Medicine, vol. 193, Article ID A4008, 2016. 
[26] N. A. Maffiuletti, A. Morelli, A. Martin et al., "Effect of gender and obesity on electrical current thresholds," Muscle and Nerve, vol. 44, no. 2, pp. 202-207, 2011.

[27] M. J. H. Sillen, C. M. Speksnijder, R.-M. A. Eterman et al., "Effects of neuromuscular electrical stimulation of muscles of ambulation in patients with chronic heart failure or COPD: a systematic review of the english-language literature," Chest, vol. 136, no. 1, pp. 44-61, 2009.

[28] I. Vivodtzev, B. Rivard, P. Gagnon et al., "Tolerance and physiological correlates of neuromuscular electrical stimulation in COPD: a pilot study," PLoS ONE, vol. 9, no. 5, Article ID e94850, 2014.

[29] A. Delitto, M. J. Strube, A. D. Shulman, and S. D. Minor, "A study of discomfort with electrical stimulation," Physical Therapy, vol. 72, no. 6, pp. 410-424, 1992.

[30] S. Feil, J. Newell, C. Minogue, and H. H. Paessler, "The effectiveness of supplementing a standard rehabilitation program with superimposed neuromuscular electrical stimulation after anterior cruciate ligament reconstruction: a prospective, randomized, single-blind study," The American Journal of Sports Medicine, vol. 39, no. 6, pp. 1238-1247, 2011. 


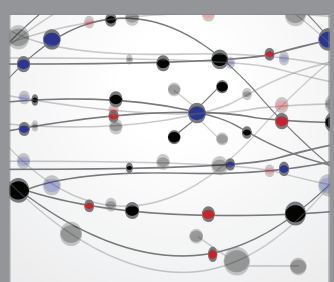

The Scientific World Journal
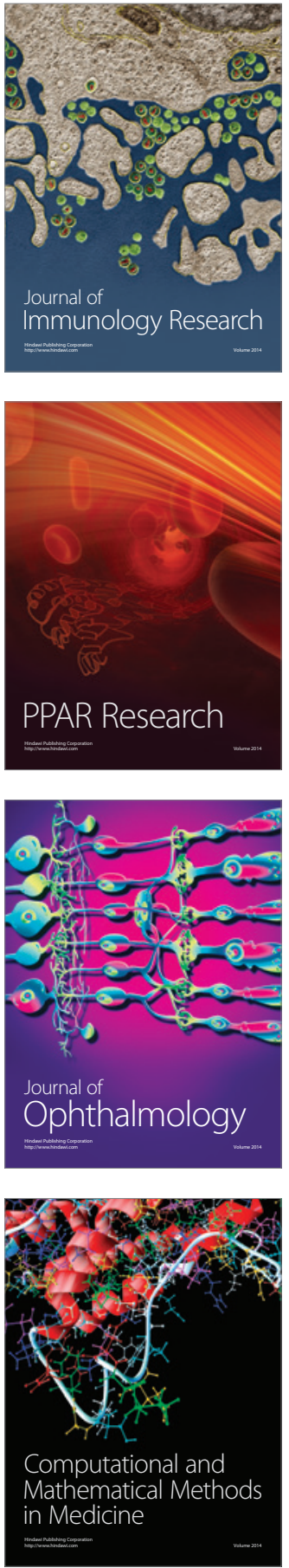

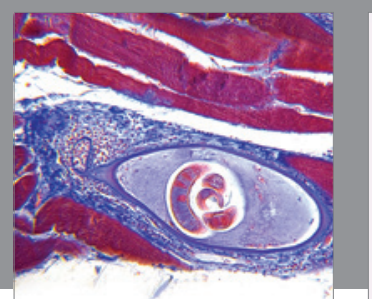

Gastroenterology Research and Practice
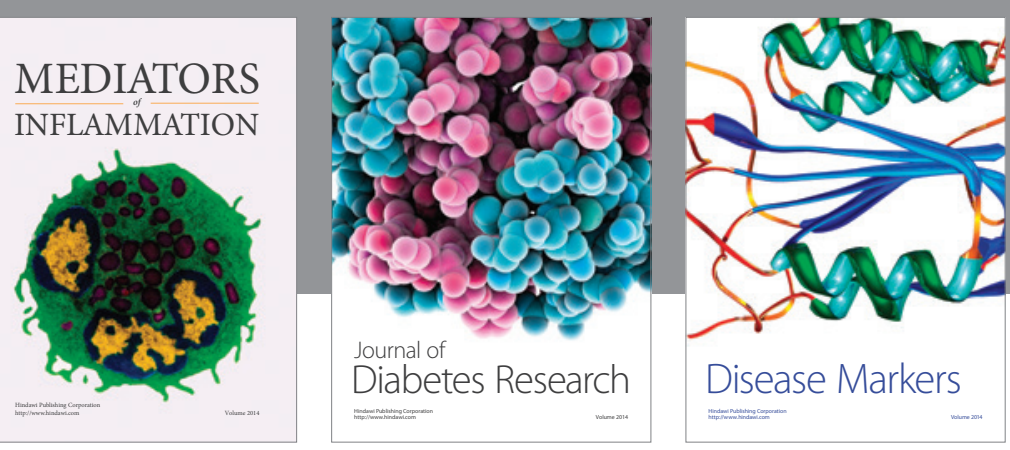

Disease Markers

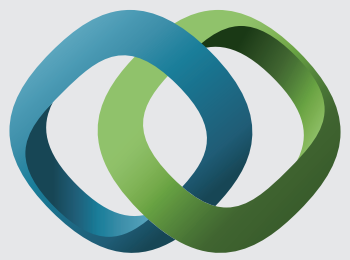

\section{Hindawi}

Submit your manuscripts at

https://www.hindawi.com
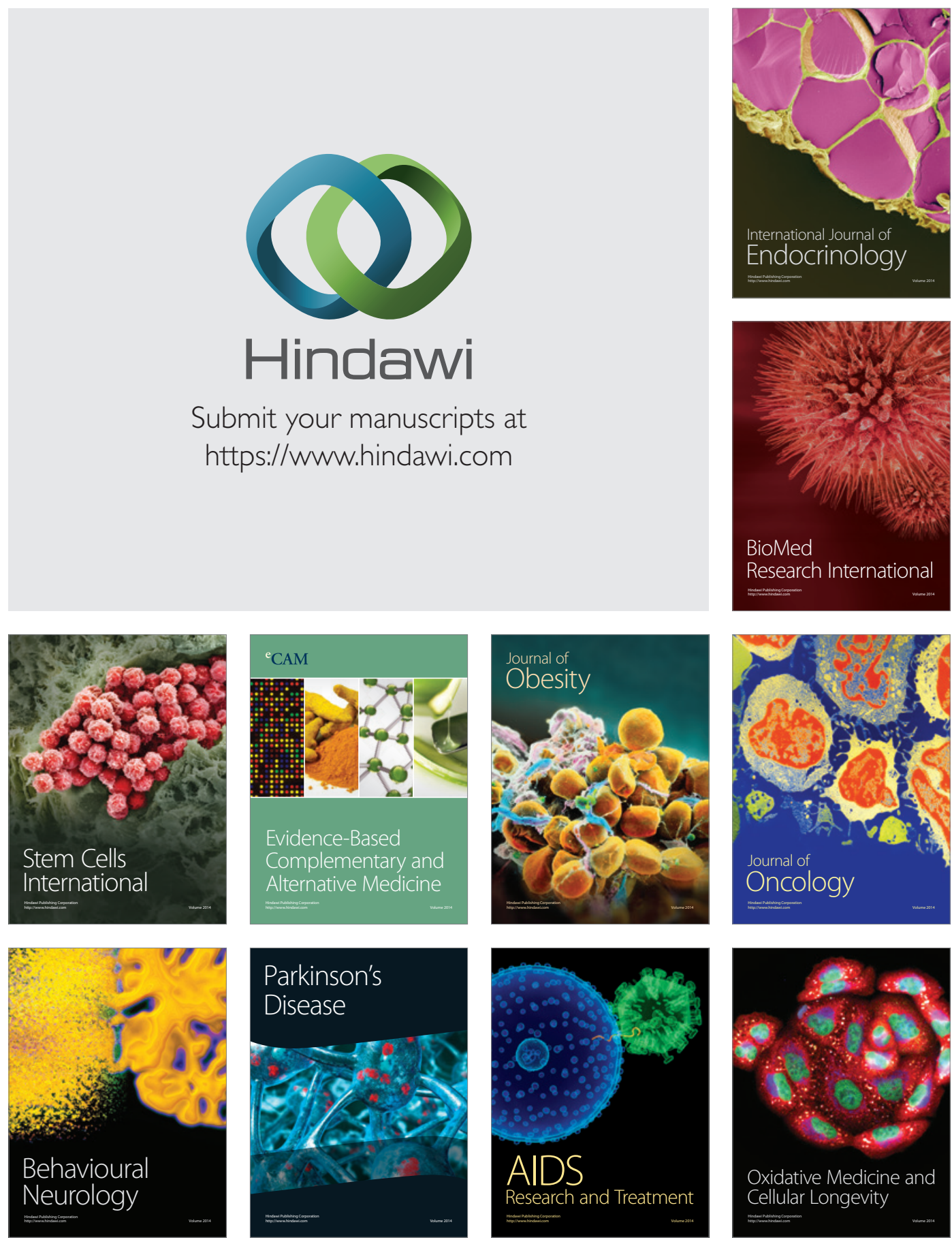